\title{
Modelos de formación inicial del profesorado de Educación Secundaria en España desde una perspectiva Europea
}

\section{Models of initial training of secondary school teachers in Spain from a European perspective}

\author{
Juan Antonio LORENZO VICENTE ${ }^{1}$, Inés María MUÑOZ GALIANO² \\ y Miguel BEAS MIRANDA ${ }^{3}$ \\ ${ }^{1}$ Universidad Complutense de Madrid, ${ }^{2}$ Universidad de Jaén \\ $\mathrm{y}^{3}$ Universidad de Granada
}

Recibido: Mayo 2014

Aceptado: Junio 2014

\begin{abstract}
Resumen
Este trabajo pretende analizar algunas claves relacionadas con los modelos de formación inicial del profesorado de Educación Secundaria desde una perspectiva internacional con referencias más específicas al modelo español. En primer lugar, realizaremos en un breve recorrido sobre la educación secundaria y sus necesidades formativas: fines, organización, estructura, carácter, duración, etc. En segundo lugar, abordaremos los modelos más característicos de la formación inicial de estos profesores: consecutivos y simultáneos, académicos y profesionales. En tercer lugar, analizaremos la estructura de la formación inicial que contiene conocimientos más generales, científicos y profesionales (con componentes teóricos y prácticos). A continuación, estableceremos una relación entre la formación inicial y el acceso a la función docente, así como la posibilidad de una titulación académica y/o profesional. En el último apartado, pondremos de relieve la cuestión de la "identidad profesional" de los futuros profesores, aspecto éste muy ligado al de la formación inicial. Por último, elaboraremos unas conclusiones finales.
\end{abstract}

Palabras clave: formación inicial, educación secundaria, identidad profesional, modelos formativos, formación científica y profesional.

\begin{abstract}
This paper seeks to analyse some key issues related to initial training models for secondary education teachers from an international perspective with more specific references to the Spanish model. First, we briefly mention secondary education and its training needs: purpose, organization, structure, character, duration, etc.. Secondly, the most typical models of initial training of these teachers: consecutive and simultaneous, academic and professional, are adressed. Thirdly, we analyse the structure of initial training containing more general scientific and professional knowledge, (its theoretical and practical components). Lastly, the relationship between initial training and access to the teaching profession will be established, as well as the possibility of academic and / or professional qualifications is analyzed. We then highlight the
\end{abstract}


issue of "professional identity", which is closely linked to the initial training of future teachers and final conclusions are discussed.

Keywords: initial training, secondary education, professional identity, educational models, scientific and professional training.

\section{Aproximación a los modelos de formación inicial de profesores de Educación Secundaria}

El trabajo que realizamos pretende analizar los modelos de formación de los profesores de Educación Secundaria y sus problemáticas desde una perspectiva europea, pero con especial referencia al modelo español. Su formación inicial ha experimentado diversas transformaciones a lo largo de los tres últimos siglos. Diversos informes (EURYDICE, 2002-2006, 2012, 2013; MEC, 2012; TALIS, 2014) han analizado las dificultades que entraña la formación de este profesorado que provienen tanto de la naturaleza de la propia Educación Secundaria, como de las transformaciones políticas, sociales, económicas, culturales y educativas sucedidas en las últimas décadas.

La comprensión y análisis del profesorado de esta etapa y de su formación inicial no puede realizarse sin tener como referencia obligada el nivel educativo en el que desarrollan sus funciones. Resulta necesario, por ello, realizar algunas reflexiones sobre su naturaleza y sus fines, estructura, organización, carácter, contenido, las funciones que tiene que desempeñar el profesorado y las nuevas necesidades formativas a las que tiene que dar respuesta. Se abordan también aspectos relacionados entre los modelos de formación inicial, su contenido y estructura, y su posible relación en el acceso a la función docente, la disyuntiva entre cursar un título académico o uno profesional y la "identidad profesional”.

Referencias sobre la Educación Secundaria y sus nuevas necesidades formativas

La Educación Secundaria tal y como ha ido considerándose desde su origen, pero especialmente en los siglos XIX y XX, época en la que nace y se consolida con identidad propia en el sistema educativo, ha ido acompañada de una serie de problemas relacionados con las funciones que se le han asignado, su organización, su estructura, carácter, contenido, duración, destinatarios, etc. (Lerena, 1980; Viñao, 1982, 1994, 2013; Sanz, 1985; Díaz, 1988; Gómez, 1996; Lorenzo, 1996, 2003 y 2011; Benso y Pereira, 2003, Egido, 2009).

Esta etapa educativa ha tenido asignados diferentes fines, según los contextos históricos a que nos refiramos, incluyendo finalmente a toda la población en su etapa obligatoria. Las tres finalidades que ha ido asumiendo históricamente la Educación Secundaria han sido: a) preparatoria (propedéutica) para determinados estudios superiores -humanísticos, científicos -; b) técnica o profesional (para determinadas profesiones de grado medio); c) terminal o finalista (formación general que se necesita para vivir en sociedad y participar en la vida social). Su organización (serial o cíclica) y estructura (un ciclo único, dos ciclos, el segundo único o con opciones o modalidades 
diferentes) también condicionan las funciones del profesorado de esta etapa educativa. Actualmente, en numerosos países se ha consolidado una organización en dos ciclos, el primero de los cuales suele ser común y el segundo diferenciado en modalidades u opciones. También puede existir un primer ciclo único o diferenciado en alguno de sus cursos y un segundo ciclo con opciones diferentes orientadas a la especialización (científico, humanístico, técnico, profesional, artístico, etc.).

Otra cuestión relacionada es el carácter de la Educación Secundaria. Nos preguntarnos si debe prevalecer su perfil formativo o el instructivo, como ha sido tradición en el caso español. Éste es un problema no menor en nuestro país, puesto que condiciona importantes decisiones sobre la formación inicial de los profesores en esta etapa. Este asunto nos lleva a poner en cuestión si ha de prevalecer en dicha formación inicial una formación académica o hay que encaminarse más bien hacia una formación profesional que incorpore ambas. En la actualidad, ya no se discute el carácter formativo de la Educación Secundaria, sobre todo en el periodo obligatorio. El problema puede plantearse en el segundo, si ha de tener una dimensión formativa, además de otras como preparatoria, profesional, etc. Respecto a la duración parece consolidada una etapa de 6 ó 7 años, que puede estar organizada en una solo ciclo o, como hemos dicho, en dos: la primera común y la segunda con opciones o modalidades diferentes. En el caso español, la Educación Secundaria con la LOE (2006) tiene una duración de 6 años y está organizada en dos etapas: Educación Secundaria Obligatoria (ESO) de 4 años y Bachillerato de 2 años, con modalidades: Artes; Ciencias y Tecnología; Humanidades y Ciencias Sociales, organizados en materias comunes, materias de modalidad y materias optativas. El Título es único (Título de Bachiller).

Actualmente la LOMCE (2013) introduce cambios en la estructura, organización y evaluación de la Educación Secundaria en España, aunque mantiene la misma duración: 6 años (4 ESO y 2 Bachillerato). La Educación Secundaria Obligatoria (art.23 bis) se organiza en materias y comprende dos ciclos, el primero en tres cursos escolares y el segundo de uno, el cual tiene un carácter fundamentalmente propedéutico. La nueva reforma educativa flexibiliza y anticipa la elección de itinerarios en tercero mediante la elección de asignaturas. Así en cuarto se podrá escoger cursar: enseñanzas académicas para la iniciación al Bachillerato o enseñanzas aplicadas para cursar la Formación Profesional. Otro de los cambios se refiere a la evaluación al finalizar las etapas de la Educación Secundaria. Así al término de cuarto de ESO, la evaluación individualizada podrá hacerse por la enseñanza cursada, permitiendo así el acceso al Bachillerato o a la Formación Profesional de grado medio. La nota obtenida tendrá un peso del 30\% frente al 70\% deducido de la media de las calificaciones de la ESO. La calificación igual o superior a 5 puntos sobre 10 permitirá la obtención del Título de Graduado en ESO. Respecto al Bachillerato (art. 34) se ofrecerán tres modalidades: Ciencias, Humanidades y Ciencias Sociales y Artes. Para la obtención del Título de Bachiller será necesaria la superación de la evaluación final de esta etapa con una calificación de 5 puntos o más. Para ello, se considerarán un $60 \%$ la media de las calificaciones obtenidas en las materias cursadas y un $40 \%$ la nota de la evaluación final. 
La propuesta surge para adaptarse a las actuales demandas de la sociedad y con la intención de que todos los estudiantes cursen la Educación Secundaria y que se atienda a su heterogeneidad. Así, se intenta evitar que la baja tasa de titulados en secundaria siga en aumento. Según publica la MEC (2012), la tasa de titulados en educación secundaria entre los 25-34 años es del 65\%, frente al 82\% de la OCDE. Igualmente el porcentaje de población entre los 20 y 24 años que ha completado al menos la Educación Secundaria superior ha crecido en Europa entre 2000 y 2010, pero en España, además de ser inferior, ha disminuido (EURYDICE, 2012).

Los Informes Internacionales y numerosos autores destacan la mayor complejidad del trabajo de los profesores de Educación Secundaria. Nuevas funciones y nuevos retos hacen que este cambio del trabajo de los profesores sea evidente: la aparición y uso de las nuevas tecnologías de la información y comunicación y la necesidad de su conocimiento y uso por parte de los profesores, sobre todo desde la perspectiva didáctica; la gestión de los centros educativos; la atención de necesidades educativas especiales, la atención a la diversidad cultural; y la necesidad de resolver problemas de comportamiento y disciplina (MEC, 2012; EURYDICE, 2012, 2013; TALIS, 2014). Es necesaria una formación de los profesores de Educación Secundaria en estos aspectos del funcionamiento real de los centros. La formación inicial de los profesores de este nivel educativo ha de incorporar estas cuestiones, además de proporcionar una buena formación científica y profesional. Las funciones actuales de los profesores de Educación Secundaria son muy diferentes a las de otras épocas y con la sola formación científica de carácter académico que se recibe en la Universidad no se puede responder adecuadamente a ellas.

\section{Modelos de formación inicial}

Desde una perspectiva histórica es necesario remontarse a los comienzos del siglo XIX cuando con la aparición de los sistemas educativos se perfilan los modelos de formación de profesores, en nuestro caso, para los Centros de Educación Secundaria (Liceos, Gymnasium, Institutos, etc.). En los inicios destacamos las tres direcciones principales que se tomaron en Europa: Francia, Prusia e Inglaterra. El caso prusiano es claro. Se optó por proporcionar la formación de los futuros profesores de Educación Secundaria en la Universidad, a través de la Facultad de Filosofía en la que proliferaron los estudios pedagógicos. Esta decisión fue acompañada muy pronto con la implantación de examen pro facultati docendi para poder ejercer de profesor de un Gymnasium, planteándose desde el principio un modelo en el que la formación científica (de especialización en una o varias disciplinas) y la formación pedagógica se recibían en la Universidad. A ello se añadía un periodo posterior de prácticas en los centros secundarios que habilitaría para el profesorado. El caso francés que terminaría con la implantación del Sistema Educativo Napoleónico, presentó al principio cierto titubeo debido a las contradicciones existentes entre las propuestas de la Revolución Francesa y la que terminó imponiéndose en la práctica: 
... inicialmente, se creó una Escuela Normal Superior, pero al poco tiempo serían las Facultades de Ciencias y de Letras, creación de la Universidad Napoleónica, las que definitivamente se terminarían encargando de la formación de los profesores de los Liceos y, consecuentemente, le acabaría dando una orientación en la que predominarían los aspectos científicos (especialización de la disciplina correspondiente) y se descuidarían los pedagógicos y prácticos (Lorenzo, 2001, p. 106).

Terminaron siendo las Facultades de Filosofía y Letras y las de Ciencias las que formaron a los profesores de los Liceos. Como señala Lorenzo (2001), en estas Facultades de nueva planta no parece que los estudios pedagógicos tuvieran sitio, por lo que se puede pensar que la formación del profesorado de los Liceos quedó pronto reducida a la especialización en las materias que tenían que impartir. Una tercera modalidad, referida a la formación del profesorado de Educación Secundaria, fue el llevado a cabo en Inglaterra (Ruiz, 1985). En este modelo cabía recibir la formación pedagógica en Universidades o en Escuelas Normales, dependientes o no de las anteriores y, sobre todo, un segundo modelo en el que sobre la formación práctica giraría el resto de la formación pedagógica.

En este inicio de la formación de los profesores de Educación Secundaria en Europa encontramos ya las claves que darán origen a los modelos que terminaron implantándose en la mayoría de los países (González, 2010). El modelo que actualmente denominamos simultáneo, el del caso prusiano, en el que la formación científica y la formación pedagógica tienen ambas un importante tratamiento; el modelo consecutivo, con matices, representado por el caso francés, en el que predomina la formación científica, quedando relegada a un papel secundario o suprimida la formación pedagógica y la formación práctica. Y el modelo inglés, más abierto, en el que una de sus direcciones le da gran importancia a la práctica docente, dejando abierta la formación pedagógica a la Universidad o a otro tipo de Centros.

Señala el Proyecto EURYDICE (2013) que la formación del profesorado incluye normalmente un componente general y otro profesional. El primero se basa en cursos que ofrecen una formación genérica y la especialización en una o varias materias. El componente profesional incluye cursos para la adquisición de aptitudes características de la profesión docente y formación práctica en centros escolares. Esta formación de carácter profesional, teórica y práctica a la vez, puede impartirse al comienzo de la educación terciaria, coincidiendo, por tanto, con la educación general (modelo simultáneo) o en una segunda fase en la que también pueden impartirse algunos cursos de carácter general (modelo consecutivo). Para cursar la formación simultánea, se requiere estar en posesión de la titulación correspondiente a la Educación Secundaria superior y en algunos casos, un certificado de aptitud para la educación terciaria. En el caso del modelo consecutivo, los estudiantes reciben una formación universitaria en un campo determinado y posteriormente cursan una formación postuniversitaria específica para el profesorado. En algunos países, la formación del profesorado finaliza con una fase de cualificación "en el lugar de trabajo" (EURYDICE, 2013). Con la información 
obtenida en el informe citado, se concreta en qué países se sigue el modelo simultáneo, el modelo consecutivo o ambos (tabla 1):

\begin{tabular}{|c|c|c|}
\hline $\begin{array}{c}\text { Modelos concurrentes } \\
\text { o simultáneos }\end{array}$ & $\begin{array}{c}\text { Modelos consecutivos } \\
\text { o sucesivos }\end{array}$ & Coexisten \\
\hline $\begin{array}{l}\text { Alemania, Bélgica, República } \\
\text { Checa, Dinamarca, Eslovaquia, } \\
\text { Estonia, Finlandia, Holanda, } \\
\text { Hungría, Letonia, Polonia, } \\
\text { Rumanía. Suecia (desde 2001) }\end{array}$ & $\begin{array}{l}\text { Bulgaria, Chipre, España, } \\
\text { Francia, Grecia, Italia, } \\
\text { Liechtenstein }\end{array}$ & $\begin{array}{l}\text { Austria, Eslovenia, Irlanda, } \\
\text { Islandia, Lituania, Noruega, } \\
\text { Reino Unido. }\end{array}$ \\
\hline
\end{tabular}

TABLA 1. Modelos de formación del profesorado de Educación Secundaria Obligatoria Fuente: EURYDICE (2013).

De los informes EURYDICE (2002-2006, 2012, 2013) se desprende una crítica al modelo consecutivo y una defensa del modelo simultáneo o concurrente, elegido mayoritariamente por los países europeos por ser más coherente con las funciones del profesorado y las finalidades de la Educación Secundaria. En esta línea, el Informe EURYDICE, (2002) señala que:

La búsqueda de una formación más orientada a la profesión está motivada por los cambios radicales que ha experimentado la Educación Secundaria en las últimas décadas, concretamente la "masificación" y los nuevos retos a los que se enfrenta el profesorado en lo que respecta a la diversidad de alumnos con los que trata. Es cada vez más habitual que el profesorado asuma un nuevo papel, que implica la adquisición de otras habilidades, además de la de enseñar una asignatura especifica... (p.45).

En el mismo sentido, se manifestaba el profesor Esteve (2003) refiriéndose a este tema de los modelos de formación de profesores de Educación Secundaria:

En toda Europa se exige a los profesores de secundaria una formación universitaria con un mínimo de cinco años de formación, si bien aún perduran las diferencias entre los modelos formativos simultáneos y sucesivos. En cualquier caso, y con independencia del modelo seguido, en todos los países se acepta ya la necesidad de una formación específica de carácter profesional y práctica, abandonando el antiguo supuesto de que para dar clases en secundaria lo único importante era dominar profundamente los contenidos científicos de una materia determinada. Los modelos sucesivos presentan el problema de crear una identidad profesional falsa en los futuros profesores de secundaria, que llegan a imaginarse a sí mismos, profesionalmente, trabajando como historiadores o como químicos, cuando en realidad van a trabajar profesionalmente como profesores de Historia o como profesores de Química (p. 211).

Resulta evidente que en el modelo simultáneo la formación científica (general y especializada) y la profesional (teórico-práctica), deben tener ambas un tratamiento importante y estar orientadas hacia la formación de un profesional de la educación. Por supuesto, debe ocupar un lugar destacado la formación práctica como colofón de ese 
proceso. Ello tiene que ver con la búsqueda de una identidad profesional que es muy difícil de conseguir en los modelos consecutivos actuales. En España, salvo algún ensayo puntual, se ha impuesto una formación inicial siguiendo el modelo consecutivo francés con predominio de una formación científica y académica, quedando muy relegada la formación profesional.

Todo indica que las reformas que se están aplicando en muchos países de Europa tratan de responder a las nuevas realidades educativas que emergen como consecuencia de los procesos globales de cambio social inducidos por el cambio de tendencias económicas y cambios colectivos en la mentalidad y los valores de los ciudadanos (MEC, 2012; EURYDICE, 2013). Las nuevas concepciones económicas, los nuevos desarrollos científicos y tecnológicos y los nuevos valores sociales hacen necesario reconsiderar el papel de los profesores de Educación Secundaria que se encuentran con una formación inicial que no les permite responder a los retos que se les presentan en la práctica. En consecuencia, se debería acentuar la dimensión profesional del profesor, sin perjuicio de la importancia que tiene su formación científica (general y especializada).

No obstante, el profesorado no coincide con esta percepción. Recientemente se ha publicado un estudio internacional referido a los datos de 2013 que examina diversos aspectos de la enseñanza y el aprendizaje a través de encuestas a los profesores y directores de centros educativos de Educación Secundaria Obligatoria de 33 países de Europa (TALIS, 2014). Se refleja en él que más del 97\% del profesorado declaran sentirse bien preparados para el trabajo docente en España. En la encuesta del MEC (2012) este porcentaje es inferior (90\%). Nos parece obvio que la percepción del profesorado sobre su preparación para dar clases y ejercer un papel eficaz en el sistema educativo, no se corresponde con la formación real de profesorado.

\section{Contenido y estructura de la formación inicial}

La formación inicial de los profesores de Educación Secundaria es, de modo generalizado, de carácter universitario. Son centros universitarios (Facultades, Departamentos, Institutos) generalmente los encargados de la formación científica (general y especializada). Existe una mayor variedad en lo que respecta a la denominada formación profesional, pues en unos casos se integra en la Universidad y en otros no. También se debe señalar que la formación general tiene un peso mayor que la formación profesional, acentuándose este hecho en el caso de los modelos consecutivos.

La duración de la formación inicial oscila entre los cuatro o cinco años (8 o 10 semestres), siendo minoritarios los países con tres años de formación o más de cinco. En el modelo simultáneo puede estar organizada la formación en un solo ciclo de estudios. En el modelo consecutivo, generalmente, son dos: el primero, más largo (4-5 años) dedicado a la formación científica; el segundo, más breve, dedicado a la formación profesional, oscilando entre varios meses y un año académico. En la mayor parte de los países, en el año 2000, el periodo mínimo de formación inicial del profesorado oscilaba entre los cuatro y los cinco años, excepto en Bélgica, Dinamarca 
e Islandia, cuya formación tenía una duración de tres años. La titulación que se obtiene al concluir la formación se corresponde con el nivel 5A, equivalente al nivel universitario convencional. Según el Informe EURYDICE (2013) sobre la formación del profesorado en lo que respecta a su contenido, la formación inicial del profesorado está formada por dos componentes:

- La formación de carácter general, que consta de cursos de naturaleza general y de la especialización en una o varias materias El objetivo de estos cursos es, por tanto, que los futuros docentes adquieran un profundo conocimiento de una o varias materias y, al mismo tiempo, una cultura general.

- La formación de carácter profesional incluye un componente práctico y otro teórico y se centran en la actividad docente. Esta etapa comprende cursos de legislación educativa, historia y sociología de la educación, psicología y metodología de la enseñanza, además de prácticas en centros escolares de corta duración, por lo general, no remuneradas, a excepción de la fase final de cualificación "en el lugar de trabajo". Los tutores de clase son los encargados de supervisar estas prácticas que el profesorado del centro de formación evalúa periódicamente. Los docentes reciben mediante esta formación de carácter general una base teórica y práctica para ejercer la profesión. La normativa existente sobre la formación inicial suele estipular la inclusión obligatoria de al menos las siguientes áreas: pedagogía, teoría de la educación, psicología, conocimiento de la materia, su didáctica y práctica docente.

En el modelo consecutivo, los estudiantes reciben inicialmente una educación de carácter general obteniendo a la finalización de sus estudios una materia o especialidad de estudios determinados. Al concluir este periodo, se matriculan en un programa de formación inicial de carácter profesional, que les permite cualificarse como profesores. El modelo simultáneo, implica la existencia de un programa que combina desde un principio la formación general con la formación teórica y práctica de carácter profesional.

La formación general ocupa la mayor parte del tiempo en ambos modelos. No obstante, en el caso del modelo simultáneo, se incrementa de modo notable la formación profesional que ronda en muchos casos el 30\% del tiempo, sobrepasando el $50 \%$ Bélgica, Alemania y Malta. Por el contrario, en los países cuya formación se corresponde con el modelo consecutivo, la proporción del componente profesional con respecto a la formación total es siempre inferior al 30\%, a excepción de Italia, Austria, Luxemburgo y Portugal. En resumen, el modelo simultáneo predomina en la formación inicial del profesorado de Educación Secundaria de primer ciclo en Europa y dedicándose más tiempo a su formación de carácter profesional que en los otros modelos (EURYDICE, 2013).

En cuanto al grado de especialización el profesorado de la primera etapa de Educación Secundaria, requiere una formación más generalista, mientras que el de la segunda, requiere un mayor grado de formación específica. Sin embargo, la excesiva especialización puede llevar consigo un riesgo de fragmentación del conocimiento no adecuado para la segunda etapa de Educación Secundaria y para el trabajo en equipo. 
Relación entre la formación inicial y el acceso a la función docente. Titulación "académica" o titulación "profesional"

Según el Informe EURYDICE (2013), son pocos los países que ofrecen un acceso libre a la formación inicial del profesorado de Educación Secundaria. Generalmente, el límite de plazas se establece a nivel central. Habitualmente, las instituciones que ofrecen la formación son las que establecen los criterios de selección o bien se toma una decisión conjunta entre éstas y las autoridades centrales. En algunos países existe una tendencia creciente a la centralización de estas decisiones, que probablemente será más marcada en los próximos años, con la introducción de medidas de control de la calidad. La preparación académica de los candidatos sigue siendo el criterio de selección más común, que se evalúa generalmente a través de los resultados obtenidos en titulaciones previas o mediante un examen de acceso. Menos frecuente es la consideración de criterios asociados a aspectos interpersonales o a la motivación por el trabajo con adolescentes. En algunos países, las instituciones de formación incluyen en sus procesos de selección una entrevista a los candidatos. La consideración de factores psicológicos y pedagógicos parece ser de gran importancia tanto para los candidatos que deciden prepararse plenamente para ejercer la profesión como para los formadores que establecen la selección y deben ofrecer una formación de calidad.

La relación entre la formación inicial y el acceso a la función docente es un problema de gran importancia tanto para los futuros profesores como para el sistema educativo y para la propia sociedad. En el caso de que exista una selección previa al acceso a la formación inicial, se está limitando la posibilidad a cuantos lo deseen y cumplan con los requisitos para ese acceso. En este caso, debería existir cierta vinculación entre la titulación alcanzada y el acceso a la función docente. Históricamente, el caso francés es notorio, al exigirles a los candidatos el compromiso de un determinado periodo de tiempo de docencia en los centros públicos. Ello implica aplicar una racionalidad pensada desde la perspectiva de las plazas de profesores que serían necesarias cubrir en los años venideros y acomodar la oferta formativa a esas expectativas, estableciéndose un "numerus clausus". En los modelos simultáneos este sistema sería más viable que en el de los consecutivos. Y en el caso de que éstos, como en España y otros países, consten de dos etapas, la limitación de plazas habría que realizarla en el segundo periodo (formación profesional). Es, sin duda, un tema complejo. Sobre este asunto señalaba el profesor Esteve (2006) que:

De forma general, la tendencia europea lleva a establecer un acceso restringido a la profesión docente frente a unos pocos países en los que todavía el acceso es libre [...] pues se considera que la profesión docente es cada vez más una profesión exigente, que exige cualificaciones cada vez más altas, y que, desde el punto de vista estratégico, el futuro del desarrollo social y económico de nuestros países en la sociedad del conocimiento depende de un alto nivel de cualificación de nuestros profesores [...] (p.28).

En este sentido la LOMCE (2013), elimina la Prueba de Acceso a la Universidad generalizada para la admisión en la enseñanza universitaria y establece en su artículo 38 que las Universidades podrán determinar la admisión del alumnado fijando diversos 
procedimientos: modalidad y materias cursadas en Bachillerato en relación con la titulación elegida, calificaciones obtenidas en materias comunes, formación académica o profesional complementaria y/o estudios superiores cursados con anterioridad. E incluso establecer evaluaciones específicas de conocimientos y/o de competencias.

Otro tema relacionado con la formación inicial y el acceso a la función docente es el que representa el dilema entre la formación para una titulación "académica" o para una titulación "profesional”. ¿Qué resulta más apropiado para la formación inicial de los futuros profesores de Educación Secundaria que ésta se organice para la obtención de un título "académico" o de un título "profesional"? En el caso de los modelos consecutivos, en muchos países, se obtiene un título "académico". El "profesional” se consigue en un segundo periodo de estudios, que suele incluir una fase de prácticas. En el caso de España, por ejemplo, la titulación profesional se alcanza después de superar unas pruebas de selección y de acceso a la función pública docente. No es el caso de los centros privados y privados-concertados, a los que se puede acceder con la formación académica y profesional recibida. Primero se forman científicos, dando lugar a profesionales vinculados a la especialización realizada. En segundo lugar y como complemento, con una duración generalmente mucho menor, se les trata de proporcionar esa formación e identidad como profesores. El profesor Esteve (1997), considera que sólo la puesta en marcha de títulos de "profesor" puede dar una respuesta satisfactoria, simultaneando la formación psicopedagógica con la formación que reciben en las Facultades, buscando, además, una formación generalista dentro de los títulos de Historia, Química, Ciencias de la Naturaleza, Física, etc.

Parece lógico pensar que el modelo simultáneo es más apropiado para organizar la formación inicial de tal modo que dé lugar a un título "profesional”. Incorporar las tres dimensiones de la formación inicial en una sola estructura organizativa facilita que la dimensión científica y la formación profesional (teórica y práctica) tengan un tratamiento adecuado y se proponga que esa formación inicial consiga profesionales de la educación. Sin embargo, en el sistema consecutivo, primero se forman científicos y, por tanto, la identidad profesional que han ido desarrollando a través de sus estudios es la de científico y no la de profesor. Ésta aparecerá como un añadido con escasas posibilidades de prevalecer sobre la anterior. Este problema nos lleva a plantear una cuestión de enorme interés: el de la "identidad profesional” (Puelles, 2009).

\section{La "identidad profesional"}

Consideramos que durante la formación inicial el futuro profesor ha de encontrar esa identidad, sin la cual le va a resultar mucho más complejo y difícil ejercer como tal. Por tanto, el modelo formativo elegido, ha de tener presente que se trata de formar profesores y no otro tipo de profesionales. Algunos países, como el caso de Alemania, por el modelo elegido, lo resolvieron pronto. España está en las antípodas de formar a sus futuros profesores para conseguir dicha identidad profesional. El modelo consecutivo adoptado, e históricamente limitado a la formación académica hasta tiempos relativamente recientes, ha conseguido que, como mucho se formen científicos, pero no profesores. Esto es algo que viene después, no tanto desde la formación como desde la experiencia profesional, al trabajar ya como profesores 
formados para otro tipo de profesiones. Se ha consolidado la creencia de que la cultura académica del profesorado, es decir, sus conocimientos disciplinares, son quienes les confieren su identidad profesional (Cuesta, Mainer y Mateos, 2010).

El profesor Esteve (2009) se muestra muy crítico con los enfoques idealizados de la formación de profesores y señala que la formación inicial hay que orientarla hacia las situaciones y problemas reales de la práctica docente, evitando así el tener que aprender del ensayo y error que tan altos costes representa, tanto para el profesor como para los estudiantes, convirtiéndose su fracaso en algo personal. Para ello propone centrar más la formación en lo que el profesor hace y en sus funciones:

[...], las actividades de formación inicial de profesores deben centrarse, en primer lugar, en enseñar al futuro profesor a analizar, con el mayor detalle posible, los múltiples factores que están influyendo en las situaciones de enseñanza en las que desarrolla su actividad docente cotidiana. Uno de los denominadores comunes de los profesores que fracasan en la enseñanza es que, como dicen los alumnos, no se enteran de dónde están, de quiénes mueven las dinámicas de la clase, de qué piensan de verdad los alumnos [...] El enfoque válido es comenzar por entender la complejidad de factores que influyen en la dinámica del aula y aprender a analizar y valorar la importancia de cada uno de estos factores para obtener el clima de clase adecuado y los resultados de aprendizaje que se desean alcanzar (p. 20).

El profesor Bolívar (2007) señala que la identidad profesional docente es el resultado de un proceso biográfico y social, dependiente de la formación inicial y de la socialización profesional en las condiciones de ejercicio de la práctica profesional. Cuando se pregunta ¿qué identidad profesional para el profesorado de secundaria?, haciendo referencia a los Informes EURYDICE (2003) y OCDE (2005) señala que la formación habitual del profesorado de Secundaria en los países europeos ha oscilado entre dos polos: el disciplinar, centrado en conseguir una maestría en el saber de una materia o disciplina, y el metodológico o pedagógico - en gran medida desvalorizado frente al primero - dirigido a proporcionar modos de enseñar, gestionar el aula y, más ampliamente educar.

La mejor formación es la que integra, desde el comienzo, ambas dimensiones. La identidad profesional, en los programas de formación inicial en España, ha sido y continúa siendo la de especialista disciplinar. Indica Esteve (2009) que, los modelos simultáneos parecen producir una identidad profesional más adecuada a la realidad, en la que el futuro profesor recibe una formación específica y práctica en todos aquellos aspectos no académicos en los que se juega el éxito o el fracaso en la enseñanza.

En España, hemos pasado del CAP, al Máster, pero como indica Antonio Viñao, cuando solo han transcurrido cuatro años de la puesta en marcha del nuevo máster, hay ya un amplio foso entre la idea o pretensiones iniciales y lo sucedido posteriormente, "se ha cambiado el nombre, pero no el modelo formativo" (Viñao, 2013, p. 31). Alejandro Tiana (2013) ha afirmado que se ha producido una reforma incompleta, entre otras razones, por la escasa interacción entre el ámbito universitario y los profesionales e instituciones destinatarias de los nuevos docentes, la insuficiente 
formación práctica del profesorado y la imposibilidad de crear un título de Grado específicamente orientado a la docencia en la Educación Secundaria. En el mismo sentido, Perales y otros (2014, p. 11), afirman que "el saber y el saber hacer como productos de la formación no conseguirán desarrollar competencias si no se utilizan en el propio contexto profesional. En el caso del profesor de educación secundaria, un contexto de aula".

Antonio Nóvoa (2009) también considera que la atención prioritaria a la práctica resulta capital para dar respuesta a la necesidad de formar buenos profesores. Su propuesta consiste en asegurar que la formación de profesores asume un fuerte componente práctico, centrado en el aprendizaje de los alumnos y en el estudio de casos concretos, teniendo siempre como referencia el trabajo escolar. Lo que implica, a su vez, una esmerada selección de centros y de profesores tutores de prácticas (Valle y Manso, 2011).

\section{A modo de conclusión}

El desarrollo de este trabajo nos permite llegar a unas conclusiones que nos facilitarán la comprensión de algunas claves del mal funcionamiento del sistema educativo español que, referido a la Educación Secundaria, muestra por ejemplo, unos altos niveles de fracaso y abandono escolar (TALIS, 2014). La propia naturaleza y estructura de la Educación Secundaria española puede ser causa de este problema, como lo son también las consecuencias de una falta de consenso político y social en materia educativa; las graves carencias formativas que existen en la formación inicial del profesorado que les impide dar respuesta a las demandas del alumnado y de la sociedad en general.

Una primera consideración final es que existe un divorcio entre los planteamientos políticos y jurídicos sobre la Educación Secundaria y su funcionamiento en la práctica. Sobre este nivel educativo cae una pesada carga heredada de su historia, permaneciendo muy vinculada a la formación universitaria (carácter propedéutico) pero alejada de su finalidad educativa.

De las diversas direcciones que en la sociedad contemporánea toma la Educación Secundaria, terminal, propedéutica, técnica y profesional, sobre todo en la segunda etapa, en España ha seguido teniendo un peso dominante la función preparatoria para los estudios universitarios en detrimento de la dimensión formativa. Tradicionalmente, se ha considerado que el Bachillerato debía tener un fin propedéutico. Esta idea sigue subsistiendo, tanto en la sociedad como, sobre todo, en gran parte de los propios profesores de Educación Secundaria. Ello conlleva una formación de carácter académico, centrada en los conocimientos que hay que conseguir, sin tener muy en cuenta otras dimensiones tanto personales como sociales a las que hay que responder. Por si fuera poco, la ampliación del derecho a la educación y de la escolaridad obligatoria hasta los dieciséis años, hace que ya no lleguen unos estudiantes previamente seleccionados, sino toda la población escolar con sus correspondientes problemáticas personales. 
Existe una tendencia a la implantación de modelos simultáneos de formación inicial de profesores de Educación Secundaria, en los que se dé cierto equilibrio entre la formación científica - general y especializada - y la formación "profesional" de carácter pedagógico y práctico. Aunque esta última tenga un peso menor, sin embargo es la base de una identidad profesional. Tanto desde el punto de vista científico como profesional se debe ir desarrollando la figura de un profesor y no de un científico o de otro tipo de profesional, que es lo que se ha hecho y se hace en la formación universitaria española. Se forman científicos y no profesores. Por tanto, pensamos que el modelo consecutivo existente en España no es el más adecuado para formar profesores de Educación Secundaria. Ello implicaría cambiar el contenido y estructura de la formación de profesores de estas etapas educativas en varias direcciones: en la propia formación científica, aumentando el peso de una formación científica general y matizando el peso de la especialización; cambiando el propio enfoque de la enseñanza universitaria, relacionando el conocimiento científico con su didáctica; introduciendo con parecido nivel de importancia la formación "profesional", a través de unos conocimientos teóricos -pedagógicos, didácticos, sociológicos, psicológicos, etc. muy asociados a la práctica docente en los que encontraría justificación y apoyo; y, desde luego, un periodo de prácticas docentes al lado de profesores experimentados y vinculada, a su vez, a la formación teórica recibida.

Un asunto muy relevante es el de la relación entre la formación inicial y el acceso a la función docente. ¿Debería implantarse un "numerus clausus" y una selección diferenciada para los futuros profesores? En España se da una importante paradoja, en la que la formación inicial tiene poco o nada que ver con el acceso a la función pública docente. Por un lado, están los requisitos para el acceso a la función pública docente: tener una licenciatura o estudios de grado en una especialidad académica y haber realizado un Máster en la especialidad a la que se quiere acceder a la función docente. Para el acceso a este Máster se requiere tener la especialidad académica o superar una prueba en la que se demuestre que se poseen los conocimientos necesarios de la misma. Por otro, la exigencia para acceder a las oposiciones es la de tener un título de Grado y el Máster sin que se especifique la especialidad, pudiendo presentarse a una plaza para la que no tiene la formación académica que sí le han exigido para acceder al Máster. No hay límite de acceso para la formación inicial, sí para el Máster, en función de las disponibilidades de la oferta universitaria, pero no están relacionadas, en ningún caso, con las necesidades del sistema educativo ni con su acceso.

La cuestión de la "identidad profesional” es un asunto pendiente de resolver, dado que con el modelo actual, se forman científicos para los que, salvo algunos casos, su pretensión no es la de ser profesores. Se pretende que con un curso posterior, en nuestro caso, el Máster, cambien su identidad inicial por la de profesor, algo que se considera muy difícil de conseguir. Proponemos que la Universidad, donde radica la formación del profesorado, forme este tipo de profesionales y no otros, lo que requiere planes diferenciados, independientemente de que se adopte un modelo simultáneo o uno consecutivo. Hay que formar profesores tanto desde la ciencia como desde la pedagogía y la práctica, lo que nos lleva a pensar que sería más conveniente establecer un "título profesional". 


\section{Referencias bibliográficas}

BENSO, M.C. y PEREIRA, M. C. (2003). El profesorado de enseñanza secundaria: retos ante el nuevo milenio. Madrid: Editorial Auria.

BOLÍVAR, A. (2007). La formación inicial del profesorado de secundaria y su identidad profesional. Estudios sobre Educación. 12, 13-30.

CUESTA, R, MAINER, J., MATEOS, J (2010). El máster de secundaria. Más allá de lo obvio.

http://www.nebraskaria.es/Nebraskaria/Trabajos_y_publicaciones_files/ARTI\%CC \%81CULO\%20MASTER\%20Versio\%CC\%81n\%20larga,\%202010.pdf.

(Consultado el 20 de junio 2014).

DÍAZ DE LA GUARDIA, E. (1988). Evolución y desarrollo de la Enseñanza Media en España. De 1875 a 1930. Un conflicto político-pedagógico. Madrid: CIDE.

EGIDO, I. (2009). La profesión docente en la Unión Europea: tipología, modelos, políticas. En Puelles, M. de.(Coord.) Profesión y vocación docente. Presente y futuro (pp. 201-227). Madrid: Biblioteca Nueva/Escuela Julián Besteiro.

ESTEVE, J.M. (1997). La formación inicial de los profesores de Secundaria. Barcelona: Editorial Ariel.

ESTEVE, J.M. (2003). La tercera revolución educativa. La educación en la sociedad del conocimiento. Barcelona: Editorial Paidós

ESTEVE, J.M. (2006). La profesión docente en Europa: perfil, tendencias y problemática. La formación inicial. Revista de Educación. 340, 19-40.

ESTEVE, J.M. (2009). La formación de profesores: Bases teóricas para el desarrollo de programas de formación inicial. Revista de Educación.350, 15-29.

EURYDICE (2002). Temas clave de la educación en Europa. Volumen 3. Informe 1: Formación inicial y transición a la vida laboral. Educación Secundaria Inferior. Madrid: Ministerio de Educación, Cultura y Deporte. https://sede.educacion.gob.es/publiventa/descargas.action?f_codigo=11393\&codigo Opcion=3. (Consultado el 4 de abril de 2013).

EURYDICE (2003). Temas clave de la educación en Europa. Volumen 3. La profesión docente en Europa. Perfil, tendencias e intereses. Informe 3: Condiciones laborales y salario. Educación Secundaria Inferior General. Madrid: Ministerio de Educación $\mathrm{y}$ Ciencia. https://sede.educacion.gob.es/publiventa/descargas.action?f_codigo=11602\&codigo Opcion=3. (Consultado el 3 de abril de 2013).

EURYDICE (2004 a). Temas clave de la educación en Europa. Volumen 3. La profesión docente en Europa: Perfil, tendencias y problemática. Informe 2: Oferta y demanda. Educación Secundaria Inferior General. Madrid: Ministerio de Educación 
https://sede.educacion.gob.es/publiventa/descargas.action?f_codigo=11733\&codigo Opcion=3. (Consultado el 3 de abril de 2013).

EURYDICE (2004 b). Temas clave de la educación en Europa. Volumen 3. La profesión docente en Europa: Perfil, tendencias y problemática. Informe 4: El atractivo de la profesión docente en el siglo XXI. Educación Secundaria Inferior General.https://sede.educacion.gob.es/publiventa/descargas.action?f_codigo=11819 \&codigoOpcion=3. (Consultado el 3 de abril de 2013).

EURYDICE (2005). Temas claves de la educación en Europa. Volumen 3. La profesión docente en Europa: Perfil, tendencias e intereses. Informe Anexo: Reformas de la Profesión Docente. Análisis histórico (1975 - 2002). Educación Secundaria Inferior General. Madrid: Ministerio de Educación y Ciencia (CIDE).

EURYDICE (2006). La evaluación de la calidad de la formación del profesorado europeo. Madrid: Ministerio de Educación y Ciencia. http://www.oei.es/docentes/articulos/evaluacion_calidad_formacion_profesorado_e uropeo_EURYDICE.pdf. (Consultado el 10/04/2013).

EURYDICE (2012). Cifras clave de la educación en Europa. Madrid: Ministerio de Educación, Cultura y Deporte. http://eacea.ec.europa.eu/education/eurydice/documents/key_data_series/134ES.pdf (Consultado el 25 de junio de 2014).

EURYDICE (2013). Key Data on Teachersad School Leaders in European. Luxembourg: Publications Office of the European Union.

GÓMEZ, Ma N. (Ed.) (1996). Pasado, presente y futuro de la Educación Secundaria en España. Sevilla: Editorial Kronos.

GONZÁLEZ, I. (coord.) (2010). El nuevo profesor de secundaria. La formación inicial del docente en el marco del Espacio Europeo de Educación Superior. Barcelona: Graó.http://dspace.unav.es/dspace/bitstream/10171/8987/1/12\%20Estudios\%20Ea.p df. (Consultado el 13/03/2014)

LERENA, C. (1980). Escuela, ideología y clases sociales en España. 2. ${ }^{a}$ ed. Barcelona: Ariel;

LEY ORGÁNICA 2/2006, de 3 de mayo de Educación (LOE). BOE de 4 de mayo de 2006.

LEY ORGÁNICA 8/2013 de 9 de diciembre, para la Mejora de la Calidad Educativa. BOE de 10 de diciembre de 2013.

LORENZO, J.A. (1996) Evolución y problemática de la Educación Secundaria Contemporánea en España. Revista Complutense de Educación. 7(2), 51-79.

LORENZO, J.A. (2001). La formación del profesorado de Enseñanza Media en España (1936-1970). Madrid: Editorial Complutense.

LORENZO, J.A. (2003). La Enseñanza Media en la España Franquista (1936-1975). Madrid: Editorial Complutense. 
LORENZO, J.A. (2011). La Educación Secundaria en España: problemática histórico educativa y actual. Participación Educativa. Número extraordinario. Profesorado de enseñanza secundaria, memoria y patrimonio, 29-47.

NÓVOA, A. (2009). Para una formación de profesores construida dentro de la profesión. Revista de Educación, 350, 203-218.

OCDE (2005). La cuestión del profesorado: atraer, capacitar y conservar a profesores eficientes. Resumen en Español. http://www.oecd.org/education/school/34991371.pdf (consultado el 13/03/2014).

MEC (2012). Panorama de la Educación 2012: Indicadores de la OCDE. http://www.mecd.gob.es/dctm/inee/internacional/panorama2012.pdf?documentId=0 901e72b81415d28. (Consultado el 3 de julio de 2014).

PERALES, F. J., CABO, J. M., VÍLCHEZ, J. M., FERNÁNDEZ-GONZÁLEZ, M., GONZÁLEZ-GARCÍA, F., JIMÉNEZ-TEJADA, P. (2014). La reforma de la formación inicial del profesorado de ciencias: propuesta de un diseño del currículo basado en competencias. Enseñanza de las Ciencias, 32 (1), 9-28

PUELLES, M. de (Coord.) (2009). Profesión y vocación docente. Presente y futuro. Madrid: Biblioteca Nueva Escuela Julián Besteiro.

RUIZ, J. (1985). La primera influencia inglesa en la formación del profesorado secundario español. En VV..AA. Actas II Congreso Nacional de Pedagogía Comparada: Las enseñanzas medias desde el ángulo comparativista (pp. 381-387). Granada: Sociedad Española de Pedagogía Comparada-UGR.

SANZ DÍAZ, F. (1985). La Segunda Enseñanza Oficial en el siglo XIX. Madrid: MEC.

TALIS (2014). Informe TALIS 2013. Estudio internacional de la enseñanza y el aprendizaje. www.mecde.gob.es/inee. (Consultado el 30 de junio de 2014).

TIANA, A. (2013). Los cambios recientes en la formación inicial del profesorado en España: una reforma incompleta. Revista Española de Educación Comparada, 22, 39-58

VALLE, J.M. y MANSO, J.(2011). La nueva formación inicial del profesorado de Educación Secundaria: modelo para la selección de buenos centros de prácticas. Revista de Educación, 354, 267-290.

VIÑAO, A. (1982). Política y Educación en los orígenes de la España Contemporánea. Madrid: Siglo XXI.

VIÑAO, A. (1994). Les origines du corps profesoral en Espagne. Les Reales Estudios de San Isidro. Paedagogica Historica International Journal of History of Education, XXX, 1994-1, 119-174.

VIÑAO, A. (2013). Modelos de formación inicial del profesorado de educación secundaria en España (siglos XIX - XXI). Revista Española de Educación Comparada, 22, 19-37. 


\section{Correspondencia con los autores}

Juan Antonio LORENZO VICENTE

Calle Miguel Servet, 7

28224 Pozuelo de Alarcón (Madrid).

e-mail: jlorenzo@edu.ucm.es

Inés María MUÑOZ GALIANO

Calle Arquitecto Pablo del Castillo García-Negrete n ${ }^{\circ} 1,1^{\circ} \mathrm{C}$

23003 Jaén

e-mail: imunoz@ujaen.es

Miguel BEAS MIRANDA

Calle Memorial Núñez Blanca, 15

18007 Granada

e-mail: mbeas@ugr.es 